\title{
Effect of omega-3 supplementation on cardiometabolic indices in diabetic patients with non-alcoholic fatty liver disease: a randomized controlled trial
}

\author{
Abbas Ali Sangouni ${ }^{1,2}$, Zahra Orang ${ }^{1,2}$ and Hassan Mozaffari-Khosravi ${ }^{1,2^{*}}$ (i)
}

\begin{abstract}
Background: Patients with non-alcoholic fatty liver disease (NAFLD) as well as type 2 diabetes mellitus (T2DM) are at increased risk for cardiovascular diseases (CVD). Omega-3 supplementation has been proposed as a possible strategy for management of cardiometabolic risk. Cardiometabolic indices can predict and evaluate the cardiometabolic risk.

Aims: We investigated the effect of omega-3 supplementation on accurate and available cardiometabolic indices including atherogenic index of plasma (AIP), Castelli risk index I, Castelli risk index II and atherogenic coefficient (AC) in diabetic patients with NAFLD.

Methods: We conducted a double-blind, randomized controlled trial (RCT) for 12 weeks. From August 2016 to March 2017, the subjects referred to Faghihi hospital in Shiraz, Iran, were recruited. Sixty diabetic patients with NAFLD were randomly assigned into the omega-3 $(2000 \mathrm{mg} / \mathrm{d}$ omega-3 capsule contained $360 \mathrm{mg} / \mathrm{d}$ eicosapentaenoic acid and $240 \mathrm{mg} / \mathrm{d}$ docosahexaenoic acid) and the placebo (liquid paraffin) groups using computer-generated random number table.

Results: Omega-3 supplementation compared to the placebo had no significant effect on AIP $(-0.11 \pm 0.20 \mathrm{vs}$. $-0.03 \pm 0.16 ; P=0.11)$, Castelli risk index I $(-0.25 \pm 0.6$ vs. $-0.07 \pm 0.7 ; P=0.42)$, Castelli risk index II $(-0.24 \pm 0.5$ vs. $-0.14 \pm 0.5 ; P=0.63)$ and $A C(-0.25 \pm 0.6$ vs. $-0.07 \pm 0.7 ; P=0.42)$. After adjusting for confounding factors, the findings remained without change.
\end{abstract}

Conclusion: Omega-3 supplementation ( $2000 \mathrm{mg} / \mathrm{d})$ for 12 weeks has no effect on cardiometabolic risk. It seems, higher doses of omega-3 can improve cordiometabolic risk. The trial was registered at Iranian Registry of Clinical Trials IRCT2016102530489N1.

Keywords: Non-alcoholic fatty liver disease, Diabetes mellitus, Omega-3, Atherogenic, Cardiometabolic

*Correspondence: mozaffari.kh@gmail.com; mozaffari@ssu.ac.ir

${ }^{2}$ Nutrition and Food Security Research Center, School of Public Health,

Shahid Sadoughi University of Medical Sciences, Yazd, Iran

Full list of author information is available at the end of the article

\begin{abstract}
Introduction
Non-alcoholic fatty liver disease (NAFLD) encompasses a range of diseases from simple steatosis to nonalcoholic steatohepatitis (NASH) [1, 2]. There is a close relationship between NAFLD and type 2 diabetes mellitus (T2DM) [3]. Insulin resistance, obesity and dyslipidemia are the main factors in the pathogenesis of NAFLD and T2DM [4-6]. The worldwide prevalence of NAFLD
\end{abstract}

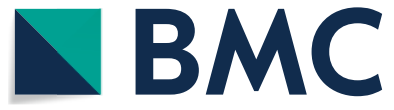

(c) The Author(s) 2021. Open Access This article is licensed under a Creative Commons Attribution 4.0 International License, which permits use, sharing, adaptation, distribution and reproduction in any medium or format, as long as you give appropriate credit to the original author(s) and the source, provide a link to the Creative Commons licence, and indicate if changes were made. The images or other third party material in this article are included in the article's Creative Commons licence, unless indicated otherwise in a credit line to the material. If material is not included in the article's Creative Commons licence and your intended use is not permitted by statutory regulation or exceeds the permitted use, you will need to obtain permission directly from the copyright holder. To view a copy of this licence, visit http://creativecommons.org/licenses/by/4.0/. The Creative Commons Public Domain Dedication waiver (http://creativeco $\mathrm{mmons}$.org/publicdomain/zero/1.0/) applies to the data made available in this article, unless otherwise stated in a credit line to the data. 
is $25.24 \%$, and the prevalence of $\mathrm{T} 2 \mathrm{DM}$ in the general population is estimated to be $6.3 \%[7,8]$. Cardiovascular disease (CVD) is one of the most common complications among patients with T2DM and NAFLD [6, 9]. Cardiometabolic indices such as atherogenic index of plasma (AIP), Castelli risk indices and atherogenic coefficient (AC) are the accurate and available tools that can predict and evaluate cardiometabolic risk [10-12]. Some recent clinical studies have used these indices to evaluate cardiometabolic risk [13, 14]. Total cholesterol (TC), triglyceride (TG), low density lipoprotein-cholesterol (LDL-c) and high density lipoprotein-cholesterol (HDL-c) are used to calculate these cardiometabolic indices [10-12].

It has been suggested that omega-3 polyunsaturated fatty acids (PUFAs), especially eicosapentaenoic acid (C20: 5n3, EPA) and docosahexaenoic acid (C22: 6n3, DHA), can improve insulin resistance, dyslipidemia and obesity have been suggested [15-17]. The experimental studies revealed that PUFAs can increase insulin sensitivity, decrease the production of very low-density lipoprotein (VLDL), and attenuate the accumulation of triglycerides in the liver [18-20]. In addition, PUFAs through modulating endothelium-relaxing, stimulating the production of gasotransmitters leading to vasorelaxation such as nitric oxide (NO) and hydrogen sulphide, and decreasing the production of endothelin 1 and angiotensin II as vasoconstricting factors can improve endothelial dysfunction and atherosclerosis $[16,21]$. Some investigations with contradictory results have investigated the effects of omega- 3 fatty acids on cardiometabolic outcomes [22-26]. The findings of these studies are inconsistent. In addition, there is no controlled trial evaluating the effect of omega- 3 fatty acids on new cardiometabolic indices such as AIP, Castelli risk indices and $\mathrm{AC}$ in diabetic patients with NAFLD. Therefore, the present study was designed to investigate the effect of omega- 3 fatty acids supplementation on cardiometabolic indices including AIP, Castelli risk index I, Castelli risk index II and AC in diabetic patients with NAFLD.

\section{Methods}

\section{Recruitment and eligibility screening}

From August 2016 to March 2017, subjects referred to the Faghihi hospital in Shiraz, Iran, were recruited and screened. Sixty patients met the inclusion criteria. The inclusion criteria were: age 18 to 65 years, diagnosis of T2DM according to the American Diabetes Association (ADA) criteria [27], and diagnosis of NAFLD by a gastroenterologist using ultrasonography (grades 1, 2 and $3)$. The exclusion criteria were: kidney diseases, type 1 diabetes, thyroid diseases, viral hepatitis, cancers, alcohol consumption, insulin infusion, pregnancy, allergy to seafood, adherence to a specific diet, poor compliance and unwillingness to continue the study.

\section{Trial design}

The present double-blind, randomized controlled trial (RCT) was conducted for 12 weeks. The participants signed a written informed consent approved by the ethical committee of Shahid Sadoughi University of Medical Sciences and Health Services, Yazd (IR.SSU.SPH. REC.1395.67). The trial was registered on 06/02/2017 at the Iranian clinical trials website under code number: IRCT2016102530489N1. The random allocation and assignment of participants into the treatment and the control groups was performed by a trained person who was not involved in the trial. Group allocation was concealed from patients and investigators. Simple (unrestricted) randomization was performed using a computer-generated random number table that was produced by a random allocation software [28]. The participants and investigators were blinded until the end of the trial.

\section{Intervention}

All omega-3 and placebo capsules were manufactured by Zahravi Pharmaceutical Company, Tabriz, Iran. The treatment group received $1000 \mathrm{mg}$ omega- 3 as gel capsule twice per day (each $1000 \mathrm{mg}$ capsule contained $180 \mathrm{mg}$ EPA and $120 \mathrm{mg}$ DHA), and the control group received the same amount of placebo (liquid paraffin) in the form of gel capsule. At the time we designed our original study, there was little evidence about the appropriate and safe dose of omega-3. The dose of omega-3 in the study of Samimi et al. [29] was $1000 \mathrm{mg} / \mathrm{d}$ (one capsule containing $180 \mathrm{mg} / \mathrm{d}$ EPA and $120 \mathrm{mg} / \mathrm{d}$ DHA). This study reported that $1000 \mathrm{mg} / \mathrm{d}$ omega 3 can improve insulin, but has no effect on lipid profile [29]. This study did not report serious adverse event related to the dosage of omega-3 [29]. We selected higher dose of omega-3 $(2000 \mathrm{mg} / \mathrm{d})$ and longer intervention duration (12 weeks) than the study of Samimi et al. [29]. The participants were asked to consume one capsule an hour before lunch and one capsule an hour before dinner. The appearance of omega-3 capsules was similar to placebo capsules. Labeling the supplement boxes was performed as A or B by a third person. The capsules were given to the participants each month. To evaluate the compliance rate, the participants were asked to deliver empty boxes of capsules to the researcher. Consuming less than $80 \%$ of expected amounts of capsules was considered the poor compliance. 


\section{Dietary intake and physical activity assessment}

At the baseline and the end of the trial, the average intake of energy as well as macronutrients was measured using a 3-day 24-h recall questionnaire. We used Nutritionist IV software (version 7.0; N-Squared Computing, Salem, OR, USA), modified for Iranian foods, to calculate intake of energy and macronutrients [30].

Physical activity can independently improve various health outcomes. In other words, significant difference between groups in physical activity can distort the real effect of intervention on outcomes. Therefore, the metabolic equivalent of task (MET) questionnaire [31] was used to assess the level of physical activity at the baseline and the end of the study.

\section{Laboratory and anthropometric evaluations}

TC, TG, LDL-c and HDL-c concentrations were measured at the baseline and the weeks 12 . After $10 \mathrm{~h}$ fasting, $10 \mathrm{ml}$ blood was collected and centrifuged. Serums were immediately frozen at $-80^{\circ} \mathrm{C}$. Levels of TC, TG, LDL-c and HDL-c were measured by an autoanalyzer (AVIDA 1800 chemistry system; Siemens, United Kingdom) using Pars Azmoon, Iran kits.

\section{Anthropometric evaluations}

Height, weight, and waist circumference (WC) were measured at the baseline and the end of the study. Assessment of height and WC was performed under the standard protocols by a measuring tape. Weight was measured by a scale (Omeron, Japan) with an accuracy of $100 \mathrm{~g}$, while the participants were with light clothes and without shoes. Body mass index (BMI) was calculated by the following formula: weight $(\mathrm{kg}) / \mathrm{height}$ squared $\left(\mathrm{m}^{2}\right)$.

\section{Cardiometabolic indices}

AIP [11], Castelli risk index I [10], Castelli risk index II [10] and AC [12] were calculated at baseline and at the end of the trial by the following equations:

$\mathrm{AIP}=\log (\mathrm{TG} / \mathrm{HDL}-\mathrm{c})[11]$.

Castelli risk index $\mathrm{I}=\mathrm{TC} / \mathrm{HDL}-\mathrm{c}[10]$.

Castelli risk index $\mathrm{II}=\mathrm{LDL}-\mathrm{c} / \mathrm{HDL}-\mathrm{c}[10]$.

$\mathrm{AC}=(\mathrm{TC}-\mathrm{HDL}-\mathrm{c}) / \mathrm{HDL}-\mathrm{c}[12]$.

\section{Sample size and statistical analysis}

In our previous article that reported the findings of insulin resistance, body composition and lipid profile [26], the sample size was estimated to be 30 for each group with 95\% confidence interval, $\alpha=0.05$, power $=80 \%$. The power analysis was performed again, and we estimated power $=80 \%$ for this article. Analysis of data was done using the Statistical Package for Social Science (SPSS) software (Chicago, Illinois, USA) version 24. To evaluate the normal distribution of variables, the Kolmogorov-Smirnov test was used. The intention-to-treat (ITT) approach was used. To compare the general characteristics between groups, we used independent $\mathrm{t}$-test and chi-square test for quantitative and qualitative variables, respectively. To compare means of variables in each group, paired t-test was used. In addition, the univariate ANCOVA was used to eliminate the effects of confounding factors. $P$-values less than 0.05 were considered the significant level.

\section{Results}

\section{Characteristics of the participants}

Sixty participants were included and with a ratio of 1:1 were randomly assigned into the treatment and the control groups. Four participants were excluded from the follow-up due to non-referral. Fifty-six participants including 28 patients in the treatment group and 28 patients in the control group completed the study (Fig. 1). At the baseline, no significant difference between the two groups was observed in baseline characteristics $(P>0.05)$; however, the intake of energy in the treatment group was significantly higher compared to the control group $(P=0.002)$ (Table 1$)$.

No significant difference was found between the two groups in the terms of weight $(P=0.61)$, BMI $(P=0.56)$ and WC $(P=0.27)$ (Table 2). Participants did not report any serious adverse effect during intervention.

\section{Outcomes}

No significant difference between the treatment group and the control group was found in the terms of AIP $(0.49 \pm 0.22$ vs. $0.44 \pm 0.19 ; P=0.36)$, Castelli risk index I ( $3.63 \pm 0.9$ vs. $3.41 \pm 0.7 ; P=0.31)$, Castelli risk index II $(2.11 \pm 0.6$ vs. $1.97 \pm 0.5 ; P=0.55)$ and $\mathrm{AC}(2.63 \pm 0.9$ vs. $2.41 \pm 0.7 ; P=0.31)$ at the baseline. In addition, there was no significant difference between the treatment and control groups in AIP $(0.38 \pm 0.28$ vs. $0.41 \pm 0.27 ; P=0.10)$, Castelli risk index I ( $3.38 \pm 0.9$ vs. $3.34 \pm 0.7 ; P=0.29)$, Castelli risk index II $(1.87 \pm 0.6$ vs. $1.83 \pm 0.5 ; P=0.47)$ and AC $(2.38 \pm 0.9$ vs. $2.34 \pm 0.7 ; P=0.29)$ at the end of the trial (Table 3).

According to mean changes, 12 -week omega-3 supplementation compared to the placebo had no significant effect on AIP $(-0.11 \pm 0.20$ vs. $-0.03 \pm 0.16 ; P=0.11)$, Castelli risk index I $(-0.25 \pm 0.6$ vs. $-0.07 \pm 0.7 ; P=0.42)$ and Castelli risk index II $(-0.24 \pm 0.5$ vs. $-0.14 \pm 0.5$; $P=0.63)$ as well as $\mathrm{AC}(-0.25 \pm 0.6$ vs. $-0.07 \pm 0.7$; 


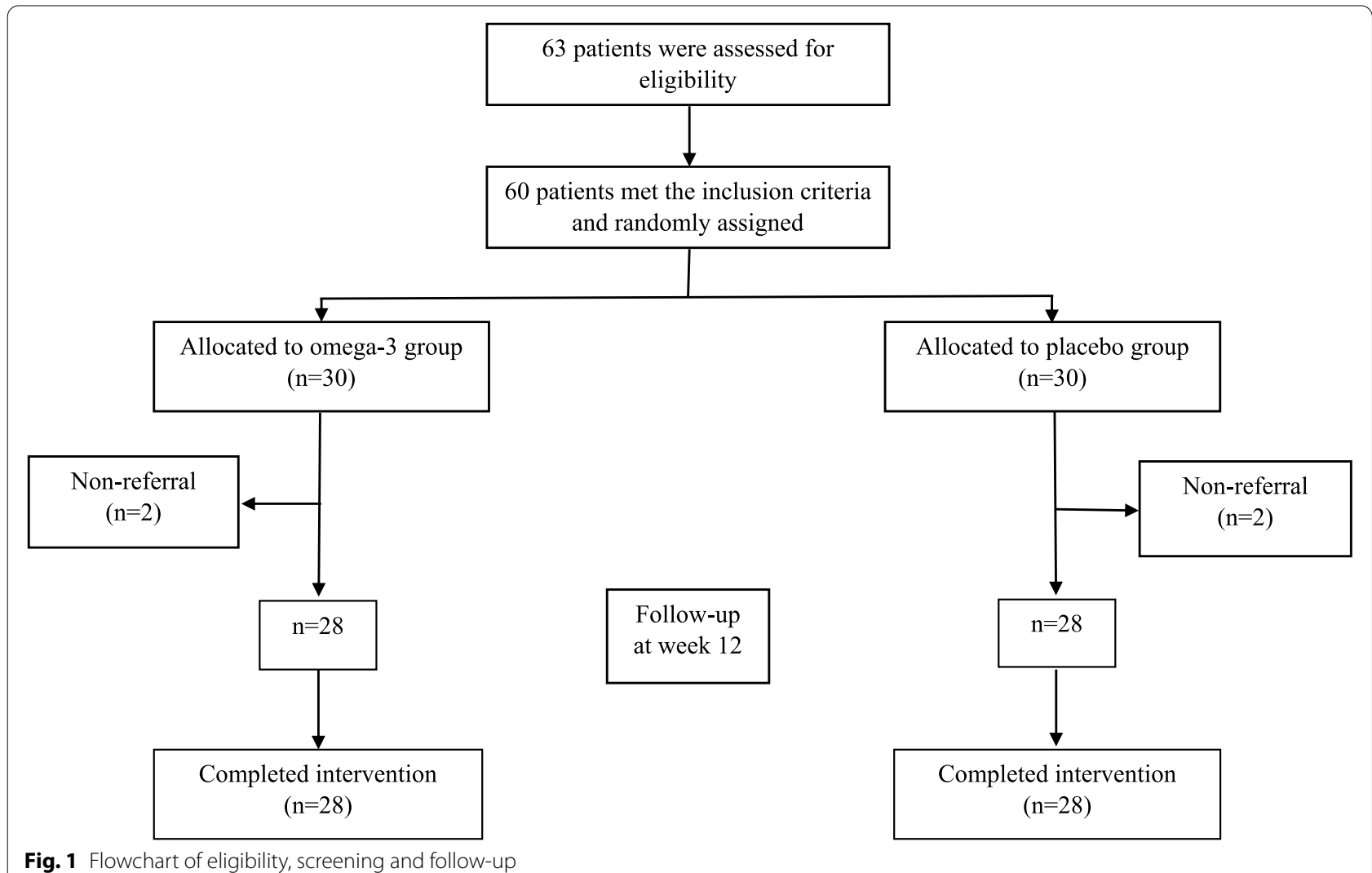

$P=0.42$ ) (Table 3). After adjusting for confounding factors, the findings remained without change.

\section{Discussion}

We demonstrated that $2000 \mathrm{mg} / \mathrm{d}$ omega-3 supplementation for 12 weeks has no effect on cardiometabolic indices including AIP, Castelli risk index I, Castelli risk index II and AC. The AIP, AC and Castelli risk indices are the accurate and available tools to assess the cardiometabolic risk [11, 12, 32, 33], and there is a direct correlate between the levels of these indices and cardiometabolic risks [11, 12, 33]. To our knowledge, the present study was the first RCT investigating the effect of omega-3 supplementation on atherogenic and Castelli risk indices in diabetic patients with NAFLD. In a randomized open-labeled trial that was conducted by Zibaeenezhad et al. [34], $2 \mathrm{~g} / \mathrm{d}$ omega-3 supplementation for 8 weeks had no effect on AIP and Castelli risk indices; but, fresh fish consumption could improve these indices. In addition to omega-3 fatty acids, fresh fish has several nutrients such as selenium, vitamin $\mathrm{D}$ and antioxidants $[35,36]$. These nutrients altogether can reduce lipid peroxidation, myocardial infarct size as well as ischemia-induced ventricular arrhythmias, and improve recovery from ischemia $[35,36]$. This can explain the greater effects of fresh fish compared to the omega-3 supplement on cardiometabolic risk. Some investigations have suggested that omega- 3 fatty acids can increase production of $\mathrm{NO}$ and hydrogen sulphide, reduce the production of vasoconstrictors such as endothelin 1 and angiotensin II, and subsequent can improve the endothelial dysfunction, reduce the platelet aggregation and blood pressure [16, 21, 37, 38]. In addition, omega-3 fatty acids through decreasing gene expression of fatty acid synthase (FAS), and phosphoenolpyruvate carboxykinase (PEPCK), reducing insulin resistance and inhibiting the inflammatory pathways triggered by cytokines can improve cardiometabolic risk [17, 21, 38-40]. The main parts of atherogenic and Castelli risk indices are serum levels of TC, TG, LDL-c and HDL-c [11, 12, 33]. Several investigations have examined the effect of omega-3 fatty acids on these variables. The study of Dasarathy et al. [24] reported that omega-3 supplementation has no effect on serum concentrations of TC, LDL-c and HDL-c. In addition, our original study demonstrated that omega- 3 supplementation for 12 weeks reduced serum TG levels, but could not improve serum concentrations of TC, LDL-c and HDL-c in diabetic patients with NAFLD [26]. Moreover, the study of Scorletti et al. [41] demonstrated that after intervention for $15-18$ months, there is no significant difference between the omega- 3 group $(4 \mathrm{~g} / \mathrm{d})$ 
Table 1 Baseline characteristics in diabetic patients with NAFLD

\begin{tabular}{|c|c|c|}
\hline & Omega-3 $(n=30)$ & Placebo $(n=30)$ \\
\hline Age $^{*}, y$ & $48.6 \pm 7.6$ & $48.8 \pm 8.7$ \\
\hline \multicolumn{3}{|l|}{ Gender* } \\
\hline Male, n (\%) & $9(30)$ & $6(20)$ \\
\hline Female, n (\%) & $21(70)$ & $24(80)$ \\
\hline Energy intake, $\mathrm{kcal} / \mathrm{d}$ & $1585.1 \pm 572.8$ & $1200.2 \pm 295.5$ \\
\hline MET, $(\mathrm{h} / \mathrm{d})$ & $28.1 \pm 4.2$ & $27.7 \pm 3.9$ \\
\hline Height* ${ }^{*}, \mathrm{~cm}$ & $160.4 \pm 8.9$ & $160.5 \pm 8.2$ \\
\hline Weight*, kg & $78.0 \pm 10.5$ & $77.1 \pm 12.5$ \\
\hline BMI $^{*}, \mathrm{~kg} / \mathrm{m}^{2}$ & $30.2 \pm 3.6$ & $29.8 \pm 4.2$ \\
\hline $\mathrm{WC}^{*}, \mathrm{~cm}$ & $106.7 \pm 9.1$ & $104.2 \pm 9.8$ \\
\hline HDL-c* ${ }^{*} \mathrm{mg} / \mathrm{dL}$ & $45.3 \pm 8.9$ & $46.5 \pm 8.03$ \\
\hline LDL-c*, mg/dL & $95.9 \pm 30.0$ & $92.2 \pm 22.2$ \\
\hline $\mathrm{TG}^{*}, \mathrm{mg} / \mathrm{dL}$ & $151.6 \pm 57.5$ & $138.4 \pm 51.8$ \\
\hline $\mathrm{TC}^{*}, \mathrm{mg} / \mathrm{dL}$ & $166.0 \pm 41.1$ & $159.9 \pm 29.3$ \\
\hline AIP* & $0.49 \pm 0.22$ & $0.44 \pm 0.19$ \\
\hline Castelli risk index $I^{*}$ & $3.63 \pm 0.9$ & $3.41 \pm 0.7$ \\
\hline Castelli risk index $\mathrm{II}^{*}$ & $2.11 \pm 0.6$ & $1.97 \pm 0.5$ \\
\hline $\mathrm{AC}^{*}$ & $2.63 \pm 0.9$ & $2.41 \pm 0.7$ \\
\hline
\end{tabular}

$P$ values are computed by independent t-test and data are expressed as mean \pm standard deviation (SD), while for Gender is computed by chi-square test and data are expressed as number (percent)

*: No significant difference was found between two groups at the baseline, except for energy intake $(P=0.002)$

NAFLD Non-alcoholic fatty liver disease, MET-h Metabolic equivalent task, BMI Body mass index, WC Waist circumference, $H D L-C$ High density lipoproteincholesterol, TG Triglyceride, TC Total cholesterol, AIP Atherogenic index of plasma, AC Atherogenic coefficient

and the placebo group in levels of TC, LDL-c and HDLc. However, the study of Zhu et al. [42] showed a significant reduction in serum LDL-c, TG after omega-3 supplementation $(6 \mathrm{~g} / \mathrm{d})$ for 24 weeks in patients with NAFLD, but the serum levels of TC and HDL-c remained without significant change. A meta-analysis on RCTs in patients with NAFLD revealed that omega-3 supplementation can reduce TG [43]. It seems, $2-4 \mathrm{~g} / \mathrm{d}$ omega-3 has a beneficial effect on TG, but not TC, LDL-c and HDLc. Omega-3 by inducing $\beta$-oxidation, decreasing VLDL synthesis, regulating gene expression of sterol-regulatory element-binding protein-1 (SREBP-1) and carbohydrate response element binding protein (ChREBP), modulating the TG synthesis, activating phosphatidic acid, diacylglycerol acyltransferase as well as LPL, and reducing the TG clearance from circulating VLDL particles can regulate serum TG [17, 21, 44-46]. To follow the principals of ethics in research, we clarify that our research group reported the effect of omega- 3 fatty acids on fatty liver, visceral adiposity index, insulin resistance, body composition and lipid profile, previously [26, 47]. We used the same data for the present article. The flowchart of eligibility, screening and follow-up as figure as well as some necessary information as table and sample size information of our previous article [26] were added to the present article.

An important strength of the present study, we measured the important confounding factors such as anthropometric variables during the follow-up. The present trial has some important limitations. We used liver ultrasonography due to its noninvasiveness and availability. Fibroscan has higher accuracy than ultrasonography in assessing severity of NAFLD $[48,49]$. Based on ultrasonography, it was possible that occur a false negative diagnosis in sampling of patients with NAFLD; however, the possible of false positive diagnosis was very low $[48,50]$. As another important limitation, we did not use cutoff point for

Table 2 Anthropometric variables in diabetic patients with NAFLD

\begin{tabular}{llll}
\hline Anthropometric variables & Omega-3 $(\mathbf{n}=\mathbf{3 0})$ & Placebo $(\mathbf{n}=\mathbf{3 0})$ & $\boldsymbol{P}^{\dagger}$ \\
\hline Weight, $\mathrm{kg}$ & & & 0.71 \\
$\quad$ Baseline & $78.0 \pm 10.5$ & $77.1 \pm 12.5$ & \\
Week 12 & $78.0 \pm 11.1$ & $76.8 \pm 13.8$ & \\
$\quad$ Mean change & $0.0 \pm 2.3$ & $-0.3 \pm 2.3$ & 0.59 \\
BMI, kg/m & & & 0.56 \\
Baseline & $30.2 \pm 3.6$ & $29.8 \pm 4.2$ & \\
Week 12 & $30.2 \pm 3.7$ & $29.6 \pm 4.5$ & 0.59 \\
Mean change & $0.0 \pm 0.8$ & $-0.2 \pm 0.8$ & \\
WC, cm & & & \\
Baseline & $106.7 \pm 9.1$ & $104.2 \pm 9.8$ & \\
Week 12 & $106.1 \pm 8.2$ & $104.8 \pm 9.6$ & \\
Mean change & $-0.7 \pm 3.7$ & $0.6 \pm 3.2$ & \\
\hline
\end{tabular}

$P$ values are computed by independent t-test and data are expressed as mean \pm standard deviation (SD)

$P$ : resulted from comparing the means of each variable at the end of the study, between two groups

$P^{\dagger}$ : resulted from comparing the mean change from baseline between groups

NAFLD Non-alcoholic fatty liver disease, BMI Body mass index, WC Waist circumference 
Table 3 Effect of omega-3 on cardiometabolic indices in diabetic patients with NAFLD

\begin{tabular}{|c|c|c|c|c|}
\hline Indices & Omega-3 $(n=30)$ & Placebo $(n=30)$ & $P^{\dagger}$ & $P^{t+}$ \\
\hline AIP & & & & 0.13 \\
\hline Baseline & $0.49 \pm 0.22$ & $0.44 \pm 0.19$ & 0.36 & \\
\hline Week 12 & $0.38 \pm 0.28$ & $0.41 \pm 0.27$ & 0.67 & \\
\hline$P$ & 0.007 & 0.33 & & \\
\hline Mean change & $-0.11 \pm 0.20$ & $-0.03 \pm 0.16$ & 0.10 & \\
\hline Castelli risk index I & & & & 0.46 \\
\hline Baseline & $3.63 \pm 0.9$ & $3.41 \pm 0.7$ & 0.31 & \\
\hline Week 12 & $3.38 \pm 0.9$ & $3.34 \pm 0.7$ & 0.88 & \\
\hline$P$ & 0.05 & 0.62 & & \\
\hline Mean change & $-0.25 \pm 0.6$ & $-0.07 \pm 0.7$ & 0.29 & \\
\hline $\begin{array}{l}\text { Castelli risk index } \\
\text { II }\end{array}$ & & & & 0.70 \\
\hline Baseline & $2.11 \pm 0.6$ & $1.97 \pm 0.5$ & 0.55 & \\
\hline Week 12 & $1.87 \pm 0.6$ & $1.83 \pm 0.5$ & 0.81 & \\
\hline$P$ & 0.01 & 0.15 & & \\
\hline Mean change & $-0.24 \pm 0.5$ & $-0.14 \pm 0.5$ & 0.47 & \\
\hline$A C$ & & & & 0.40 \\
\hline Baseline & $2.63 \pm 0.9$ & $2.41 \pm 0.7$ & 0.31 & \\
\hline Week 12 & $2.38 \pm 0.9$ & $2.34 \pm 0.7$ & 0.88 & \\
\hline$P$ & 0.05 & 0.62 & & \\
\hline Mean change & $-0.25 \pm 0.6$ & $-0.07 \pm 0.7$ & 0.29 & \\
\hline
\end{tabular}

$P$ values are computed by independent t-test and data are expressed as mean \pm standard deviation (SD)

$P$ : resulted from comparisons within groups by paired t-test

$P^{\dagger}$ : resulted from comparisons between two groups by independent t-test

$P^{\dagger \dagger}$ : resulted from comparing the mean change from baseline between groups using univariate ANCOVA after adjusting for energy intake and weight change NAFLD Non-alcoholic fatty liver disease, AIP Atherogenic index of plasma, $A C$ Atherogenic coefficient

alanine aminotransferase (ALT) levels in inclusion criteria. In addition, we used low dose of omega-3, and this is another important limitation of this study.

\section{Conclusion}

Omega-3 supplementation $(2000 \mathrm{mg} / \mathrm{d})$ has no effect on cardiometabolic indices such as AIP, Castelli risk index I, Castelli risk index II and AC. Further studies with longer intervention durations and higher doses of omega- 3 are still required to clarify our vision.

\section{Abbreviations}

AC: Atherogenic coefficient; ADA: American Diabetes Association; AIP: Atherogenic index of plasma; ALT: Alanine aminotransferase; BMI: Body mass index; ChREBP: Carbohydrate-responsive element-binding protein; DHA: Docosahexaenoic acid; EPA: Eicosapentaenoic acid; FAS: Fatty acid synthase; HDL-c: High-density lipoprotein cholesterol; ITT: Intention-to-treat; LDL-c: Low-density lipoprotein cholesterol; LPL: Lipoprotein lipase; NAFLD: Non-alcoholic fatty liver disease; MET: Metabolic equivalent of task; PEPCK: Phosphoenolpyruvate carboxykinase; RCT: Randomized controlled trial; SPSS: Statistical Package for Social Science; SREBP-1: Sterol-regulatory element-binding protein-1; No: Nitric oxide;
T2DM: Type 2 diabetes mellitus; TC: Total cholesterol; TG: Triglyceride; VAI: Visceral adiposity index; VLDL: Very low-density lipoprotein; WC: Waist circumference.

\section{Acknowledgments}

We acknowledge the contribution of the patients and co-researchers.

\section{Authors' contributions}

H.M-K: designed the research; Z.O: conducted the research; A.S: analyzed the data and wrote the manuscript; H.M-K: critically revised the manuscript. All of the authors read and approved the final manuscript.

\section{Funding}

Shahid Sadoughi University of Medical Sciences and Health Services, Yazd, Iran supported this study. This was a financial support for student thesis process including laboratory works, and provide kits as well as supplements.

\section{Availability of data and materials}

The datasets used and/or analyzed during the current study available from the corresponding author on reasonable request.

\section{Declarations}

\section{Ethics approval and consent to participate}

The study protocol was approved by Shahid Sadoughi University of Medical Sciences and Health Services. In addition, the written informed consent, which was obtained from all subjects before the data collection, was approved by the ethical committee of Shahid Sadoughi University of Medical Sciences and Health Services in Yazd, under code number: IR.SSU.SPH.REC.1395.67. All methods were performed in accordance with the Helsinki Declaration.

\section{Consent for publication}

Not applicable.

\section{Competing interests}

The authors have declared no competing interests.

\section{Author details}

${ }^{1}$ Department of Nutrition, School of Public Health, Shahid Sadoughi University of Medical Sciences, Yazd, Iran. ${ }^{2}$ Nutrition and Food Security Research Center, School of Public Health, Shahid Sadoughi University of Medical Sciences, Yazd, Iran.

Received: 6 May 2021 Accepted: 1 December 2021

Published online: 15 December 2021

\section{References}

1. Polyzos SA, Mantzoros CS. Nonalcoholic fatty future disease. Metab Clin Exp. 2016;65(8):1007-16.

2. Chalasani N, Younossi Z, Lavine JE, Diehl AM, Brunt EM, Cusi K, et al. The diagnosis and management of non-alcoholic fatty liver disease: practice guideline by the American Association for the Study of Liver Diseases, American College of Gastroenterology, and the American Gastroenterological Association. Hepatology. 2012;55(6):2005-23.

3. Hazlehurst JM, Woods C, Marjot T, Cobbold JF, Tomlinson JW. Non-alcoholic fatty liver disease and diabetes. Metabolism. 2016:65(8):1096-108.

4. Sangouni AA, Ghavamzadeh S, Jamalzehi A. A narrative review on effects of vitamin D on main risk factors and severity of non-alcoholic fatty liver disease. Diabetes Metab Syndr Clin Res Rev. 2019;13(3):2260-5.

5. Laville M, Nazare JA. Diabetes, insulin resistance and sugars. Obes Rev. 2009;10(Suppl 1):24-33.

6. Tkáč I. Metabolic syndrome in relationship to type 2 diabetes and atherosclerosis. Diabetes Res Clin Pract. 2005;68(Suppl 1):S2-9.

7. Araújo AR, Rosso N, Bedogni G, Tiribelli C, Bellentani S. Global epidemiology of non-alcoholic fatty liver disease/non-alcoholic steatohepatitis: what we need in the future. Liver Int. 2018;38(Suppl 1):47-51.

8. Abdul M, Khan B, Hashim MJ, King JK, Govender RD, Mustafa H, et al. Epidemiology of type 2 Diabetes - global burden of disease and forecasted trends. J Epidemiol Glob Health. 2020;10:107-11. 
9. Gaggini M, Morelli M, Buzzigoli E, DeFronzo RA, Bugianesi E, Gastaldelli A. Non-alcoholic fatty liver disease (NAFLD) and its connection with insulin resistance, dyslipidemia, atherosclerosis and coronary heart disease. Nutrients. 2013;5(5):1544-60.

10. Koca TT, Tugan CB, Seyithanoglu M, Kocyigit BF. The clinical importance of the plasma atherogenic index, other lipid indexes, and urinary sodium and potassium excretion in patients with stroke. Eurasian J Med. 2019;51(2):171-5.

11. Fernández-Macías JC, Ochoa-Martínez AC, Varela-Silva JA, Pérez-Maldonado IN. Atherogenic index of plasma: novel predictive biomarker for cardiovascular illnesses. Arch Med Res. 2019;50(5):285-94

12. Sujatha R, Kavitha S. Atherogenic indices in stroke patients: a retrospective study. Iran J Neurol. 2017:16(2):78-82.

13. Sangouni AA, Sasanfar B, Ghadiri-Anari A, Hosseinzadeh M. Effect of L-carnitine supplementation on liver fat content and cardiometabolic indices in overweight/obese women with polycystic ovary syndrome: a randomized controlled trial. Clin Nutr ESPEN. https://doi.org/10.1016/j.clnesp.2021.08. 005.

14. Izadi A, Shirazi S, Taghizadeh S, Gargari BP. Independent and additive effects of coenzyme Q10 and vitamin E on Cardiometabolic outcomes and visceral adiposity in women with polycystic ovary syndrome. Arch Med Res. 2019:50(2):1-10.

15. Lombardo YB, Chicco AG. Effects of dietary polyunsaturated $n-3$ fatty acids on dyslipidemia and insulin resistance in rodents and humans. A review. J Nutr Biochem. 2006;17(1):1-13.

16. Kromhout D, Yasuda S, Geleijnse JM, Shimokawa H. Fish oil and omega-3 fatty acids in cardiovascular disease: do they really work? Eur Heart t. 2012;33(4):436-43.

17. Albracht-Schulte K, Kalupahana NS, Ramalingam L, Wang S, Rahman SM Robert-McComb J, et al. Omega-3 fatty acids in obesity and metabolic syndrome: a mechanistic update. J Nutr Biochem. 2017;2018(58):1-16.

18. Alwayn IPJ, Andersson C, Zauscher B, Gura K, Nosé V, Puder M. Omega-3 fatty acids improve hepatic steatosis in a murine model: potential implications for the marginal steatotic liver donor. Transplantation. 2005;79(5):606-8.

19. Alwayn IPJ, Gura K, Nosé V, Zausche B, Javid P, Garza J, et al. Omega-3 fatty acid supplementation prevents hepatic steatosis in a murine model of nonalcoholic fatty liver disease. Pediatr Res. 2005;57(3):445-52.

20. Di Minno MND, Russolillo A, Lupoli R, Ambrosino P, Di Minno A, Tarantino G. Omega-3 fatty acids for the treatment of non-alcoholic fatty liver disease. World J Gastroenterol. 2012;18(41):5839-47.

21. Davidson MH. Mechanisms for the Hypotriglyceridemic effect of marine Omega-3 fatty acids. Am J Cardiol. 2006;98(4 SUPPL.1):27-33.

22. Capanni M, Calella F, Biagini MR, Genise S, Raimondi L, Bedogni G, et al. Prolonged $\mathrm{n}-3$ polyunsaturated fatty acid supplementation ameliorates hepatic steatosis in patients with non-alcoholic fatty liver disease: a pilot study. Aliment Pharmacol Ther. 2006;23(8):1 143-51.

23. Crestani DM, Bonin ÉFR, Barbieri RA, Zagatto AM, Higino WP, Milioni F. Chronic supplementation of omega-3 can improve body composition and maximal strength, but does not change the resistance to neuromuscular fatigue. Sport Sci Health. 2017;13(2):259-65.

24. Dasarathy S, Dasarathy J, Khiyami A, Yerian L, Hawkins C, Sargent R, et al. Double-blind randomized placebo-controlled clinical trial of omega 3 fatty acids for the treatment of diabetic patients with nonalcoholic steatohepatitis. J Clin Gastroenterol. 2015;49(2):137-44.

25. Orang Z, Mohsenpour MA, Mozaffari-Khosravi H. Effect of Omega-3 fatty acid supplementation on inflammatory markers and insulin resistance indices in patient with type 2 diabetes and nonalcoholic fatty liver: a randomized double-blind clinical trial. Obes Med. 2020;19(July):100278.

26. Orang Z, Mozaffari-Khosravi H, Sivandzadeh G, Pantovic A. The effect of omega-3 supplementation on glycemic indices and lipid profile in type 2 diabetic patients with non-alcoholic fatty liver disease: a double-blind, randomized, clinical trial. Med J Nutrition Metab. 2019;12(2):187-96.

27. Diabetes AA. Diagnosis and classification of diabetes mellitus. Diabetes Care. 2010;36(Suppl 1):S67-74.

28. Saghaei M. Random allocation software for parallel group randomized trials. BMC Med Res Methodol. 2004;4:26.

29. Samimi M, Jamilian M, Asemi Z, Esmaillzadeh A. Effects of omega-3 fatty acid supplementation on insulin metabolism and lipid profiles in gestational diabetes: randomized, double-blind, placebo-controlled trial. Clin Nutr. 2015:34(3):388-93.
30. Pehrsson PR, Haytowitz DB, Holden JM, Perry CR, Beckler DG. USDA's national food and nutrient analysis program: food sampling. J Food Compos Anal. 2000;13(4):379-89.

31. Ainsworth BE, Haskell WL, Whitt MC, Irwin ML, Swartz AM, Strath SJ, et al. Compendium of physical activities: an update of activity codes and MET intensities. Med Sci Sports Exerc. 2000;32(9 SUPPL):S498-504.

32. Dobiášová M, Frohlich J, Šedová M, Cheung MC, Brown BG. Cholesterol esterification and atherogenic index of plasma correlate with lipoprotein size and findings on coronary angiography. J Lipid Res. 2011;52(3):566-71.

33. Singh M, Pathak MS, Paul A. A study on atherogenic indices of pregnancy induced hypertension patients as compared to normal pregnant women. J Clin Diagn Res. 2015;9(7):BC05-8.

34. Zibaeenezhad MJ, Ghavipisheh M, Attar A, Aslani A. Comparison of the effect of omega-3 supplements and fresh fish on lipid profile: a randomized, open-labeled trial. Nutr Diabetes. 2017;7(12):1-8.

35. Mozaffarian D. Fish, mercury, selenium and cardiovascular risk: current evidence and unanswered questions. Int J Environ Res Public Health. 2009;6(6):1894-916.

36. Brown K, Arthur J. Selenium, selenoproteins and human health: a review. Public Health Nutr. 2001:4(2b):593-9.

37. McEwen BJ, Morel-Kopp MC, Tofler GH, Ward CM. The effect of omega-3 polyunsaturated fatty acids on fibrin and thrombin generation in healthy subjects and subjects with cardiovascular disease. Semin Thromb Hemost. 2015:41(3):315-22.

38. DeFilippis AP, Blaha MJ, Jacobson TA. Omega-3 fatty acids for cardiovascular disease prevention. Curr Treat Options Cardiovasc Med. 2010;12(4):365-80.

39. Calder PC. Omega-3 fatty acids and inflammatory processes. Nutrients. 2010;2(3):355-74.

40. Pachikian BD, Essaghir A, Demoulin JB, Neyrinck AM, Catry E, de Backer FC, et al. Hepatic n-3 polyunsaturated fatty acid depletion promotes steatosis and insulin resistance in mice: genomic analysis of cellular targets. PLoS One. 2011;6(8):e23365.

41. Scorletti E, Bhatia L, Mccormick KG, Clough GF, Nash K, Hodson L, et al. Effects of purified eicosapentaenoic and docosahexaenoic acids in nonalcoholic fatty liver disease: results from the WELCOME* study. Hepatology. 2014;60(4):1211-21.

42. Zhu FS, Liu S, Chen XM, Huang ZG, Zhang DW. Effects of n-3 polyunsaturated fatty acids from seal oils on nonalcoholic fatty liver disease associated with hyperlipidemia. World J Gastroenterol. 2008;14(41):6395-400.

43. He XX, Wu XL, Chen RP, Chen C, Liu XG, Wu BJ, et al. Effectiveness of omega-3 polyunsaturated fatty acids in non-alcoholic fatty liver disease: a meta-analysis of randomized controlled trials. PLoS One. 2016;11(10):1-22.

44. Kim HJ, Takahashi M, Ezaki O. Fish oil feeding decreases mature sterol regulatory element-binding protein 1 (SREBP-1) by down-regulation of SREBP-1 $\mathrm{c}$ mRNA in mouse liver. A possible mechanism for down-regulation of lipogenic enzyme mRNAs. J Biol Chem. 1999;274(36):25892-8.

45. Khan S, Minihane AM, Talmud PJ, Wright JW, Murphy MC, Williams CM, et al. Dietary long-chain n-3 PUFAs increase LPL gene expression in adipose tissue of subjects with an atherogenic lipoprotein phenotype. J Lipid Res. 2002;43(6):979-85.

46. Harris WS, Bulchandani D. Why do omega-3 fatty acids lower serum triglycerides? Curr Opin Lipidol. 2006;17(4):387-93.

47. Sangouni AA, Orang Z, Mozaffari-Khosravi H. Effect of omega-3 supplementation on fatty liver and visceral adiposity indices in diabetic patients with non-alcoholic fatty liver disease: a randomized controlled trial. Clin Nutr ESPEN. 2021:44:130-5.

48. Hernaez R, Lazo M, Bonekamp S, Kamel I, Brancati FL, Guallar E, et al. Diagnostic accuracy and reliability of ultrasonography for the detection of fatty liver: a meta-analysis. Hepatology. 2011;54(3):1082-90.

49. Eddowes PJ, Sasso M, Allison M, Tsochatzis E, Anstee QM, Sheridan D, et al. Accuracy of FibroScan controlled attenuation parameter and liver stiffness measurement in assessing Steatosis and fibrosis in patients with nonalcoholic fatty liver disease. Gastroenterology. 2019;156(6):1717-30.

50. Saleh HA, Abu-Rashed AH. Liver biopsy remains the gold standard for evaluation of chronic hepatitis and fibrosis. J Gastrointestin Liver Dis. 2007;16(4):425-6.

\section{Publisher's Note}

Springer Nature remains neutral with regard to jurisdictional claims in published maps and institutional affiliations. 